\title{
LA IMPORTANCIA DE LA CONSTRUCCIÓN DE LAS RELACIONES ENTRE NIÑOS, NIÑAS Y ADULTOS PARA CRECER LIBRES. LA POSTURA DE LA EDUCACIÓN LIBRE EN EL CONTEXTO DE CATALUÑA
}

\author{
Valeria Vittoria Aurora Bosna ${ }^{1}$
}

\begin{abstract}
Resumen: El presente trabajo toma en examen la importancia de la construcción y el desarrollo de las relaciones entre nińos, nińas y adultos para favorecer un verdadero crecimiento libre. Objetivo del estudio es presentar el movimiento de la Educación Libre, contextualizándolo a la región de Cataluña, y sus prácticas educativas. Estas últimas se fundamentan en la instauración de relaciones y vínculos personales entre los protagonistas del proceso educativo y van más allá de las relaciones clásicas entre estudiantes y maestros o padres. En efecto fundan espacios simbólicos únicos en que la libertad de ser se realiza verdaderamente. El marco teórico elegido es lo de la Educación Libre. Esta particular mirada pedagógica forma parte de la educación alternativa, consistente en un modelo educativo que va más allá de las metodologías tradicionales adoptadas por los sistemas formales; se inspira más bien con propuestas de educación no formal, es una oferta educativa poco masificada, y acoge diversas miradas, intereses, peculiaridades y prácticas diferentes; todo con el eje de base de educar para crecer, aprender y formarse en libertad.
\end{abstract}

Palabras clave: Educación Libre, Educación alternativa, Relación educativa.

1 Investigadora externa del Grupo de Investigación y Asesoramiento Didáctico - Facultad de Educación - Universitat de Barcelona. Licenciada con matrícula de honor en Ciencias de la Educación - Universidad de Bari (Italia). Doctora en Pedagogía Intercultural - Universidad de Messina (Italia). Máster en Estudios Interdisciplinares de Género - Universidad Autónoma de Madrid. Doctora en Pedagogía y Ciencias de la Educación en la Universidad de Foggia (Italia) y en Educación y Sociedad en la Universitat de Barcelona. Máster en Estudios de la Diferencia Sexual - Universitat de Barcelona, Máster en Sviluppo umano e Ambiente - Università Cattolica (Brescia - Italia). Temas de investigación: Historia de la Educación femenina, Pedagogía de Género, Escultismo femenino y Guidismo, Educación Libre, Prácticas educativas de empoderamiento para las mujeres, Infancia. 


\title{
THE IMPORTANCE OF BUILDING RELATIONSHIPS BETWEEN CHILDREN AND ADULTS TO GROW IN FREEDOM. THE ACTITUD OF FREE EDUCATION IN THE CATALAN CONTEXT
}

\begin{abstract}
The present work takes into account the importance of building and developing relationships between children and adults to promote a true free growth. The aim of the study is to present the Free Education movement, contextualizing it in the region of Catalonia, and its educational practices. These are based on the establishment of relationships and personal links between the protagonists of the educational process and go beyond the classical relations between students and teachers or parents. As a matter of fact, they build unique symbolic spaces in which the freedom of being can be truly realized. The chosen theoretical framework is that one of Free Education. This particular pedagogical view is part of alternative education, consisting of an educational model that goes beyond the traditional methodologies adopted by formal systems; it is rather inspired by proposals of nonformal education, it's a little overcrowded educational offer, and it hosts different views, interests, peculiarities and practices; all with the basic axis of education to grow, learn and train in freedom.

Keywords: Free education, alternative education, educational relationship.

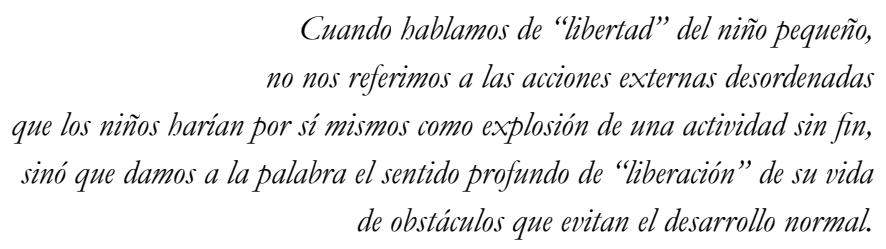

(Montessori: 1999, p.40)

\section{Introducción}

El presente trabajo de investigación ${ }^{2}$ toma en examen la importancia de la construcción y el desarrollo de las relaciones entre niños, niñas y adultos para favorecer un verdadero crecimiento libre.

Objetivo del estudio es, entonces, presentar el movimiento de la Educación Libre, contextualizándolo a la región de Cataluña ${ }^{3}$, y sus prácticas educativas, analizadas a través de un cuestionario. Estas últimas se fundamentan en la

2 Este trabajo ha sido sacado por un estudio académico más amplio contenido en la tesis doctoral con titulo La libertad femenina en Educación. Prácticas, protagonistas, ideas, desarrollada, por la misma autora del presente artículo, en Co tutela entre las Universidades de Barcelona y de Foggia (Italia) en los años 2011-2015.

3 Los centros de Educación Libre alcanzados en Cataluña son 40 (en la provincia de Barcelona 31; en Girona 5, en Lleida 1 y en Tarragona 3). Este dato no asegura la totalidad de las realidades existentes en el territorio catalán. El número de los centros, durante la investigación, ha cambiado sea con respecto a su aumento, así como a su disminución. Para esta investigación se han tomado en cuenta los centros nacidos hasta al 2014.

El conjunto de los centros es la muestra de análisis de la investigación, en particular los 20 centros que han contestado al cuestionario, herramienta metodológica principal de esta investigación. 
instauración de relaciones y vínculos personales entre los protagonistas del proceso educativo (niños, niñas, educadores, educadoras, madres y padres) y van más allá de las relaciones clásicas entre estudiantes y maestros o padres, en efecto fundan espacios simbólicos únicos en que la libertad de ser se realiza verdaderamente.

El marco teórico elegido es lo de la Educación Libre. Esta particular mirada pedagógica forma parte de la denominada educación alternativa, consistente en un modelo educativo que va más allá de las metodologías tradicionales adoptadas por los sistemas formales ${ }^{4}$; se inspira más bien con propuestas de educación no formal, es una oferta educativa poco masificada, y acoge diversas miradas, intereses, peculiaridades y prácticas diferentes; todo con el eje de base de educar para crecer, aprender y formarse en libertad.

Aunque podría considerarse que la Educación Libre aglutina un modelo educativo, la realidad es que no existe un modelo estructurado y codificado, sinó que más bien rechaza la posibilidad de ser cerrado en una categoría o modelo bien definido, y por esta razón quizás es más correcto hablar de "experiencias" de Educación Libre. Bajo esta denominación forman parte todas las realidades educativas que se caracterizan por el trabajo en libertad.

La elección de la Educación Libre parte desde la consideración que en el nuevo siglo XXI sea necesario pensar a un nuevo paradigma educativo, a nuevas metodologías e a una manera renovada de pensar y hacer educación libre de las tendencias socio-económicas que en el XX siglo han fuertemente orientado los objetivos educativos, especialmente, pero no solo, del sistema educativo formal, hacia la constitución de sujetos "educados" a las normas del mundo laboral y a sus implacables lógicas de provecho.

"Es una especie de ley no escrita que la escuela constituye la voz más autoritaria para dar opiniones con respecto a los niños e que posee en exclusiva la capacidad de proponer una educación impecable"(Wild: 2011, p.34); pero es también verdad que desde varias partes llegan señales de una profunda crisis del sistema educativo formal que vive una incoherencia entre las expectativas que crea en los estudiantes, una oferta educativa que punta a la adquisición de títulos de estudio siempre más altos, el aprendizaje de contenidos y competencias orientadas a la realización laboral en ámbitos sociales elevados, y el desatender el cuidado de las relaciones educativas, la educación a las emociones y sobretodo la creación de una positiva y equilibrada relación con sí mismo y con los demás. Una incoherencia que comprende también los educadores, las educadoras y los docentes que son siempre más orientados a conducir las nuevas generaciones más bien que a acompañarlas en el descubrimiento de las propias potencialidades y capacidades de auto desarrollo y auto educación.

Los y las profesionales de la educación, y también los adultos en general, son importantes responsables de la mediación entre el mundo y el desarrollo de los y las jóvenes y tienen el deber de reflexionar en las consecuencias de la tensión emocional

4 La educación formal se refiere a la educación aprobada por el Estado. 
negativa que el sistema educativo formal, hoy en día, pone en los estudiantes pidiendo excelencia pero tomando medida de tal excelencia en estándares creados sobre individuos imaginarios y no sobre criterios individuales, diferentes para cada uno o una de ellos.

Desde estas consideraciones nace una análisis critica hacia un sistema que impone criterios sobre lo que dice ser justo y necesario para un buen crecimiento de niños y niñas y no deja a ellos la libertad de experimentarse en procesos personales de autonomía, a ese propósito dice Rebeca Wild": "el programa de estudios oculto permanece intacto. Se trata de un programa que no admite alguna protesta: la escuela educa nuestros hijos a obedecer, a la puntualidad y al trabajo rutinario"(Wild: 2011, p.36).

Este recorrido resulta estresante y no cuida verdaderamente el crecimiento personal y la formación de una conciencia de sí, no valoriza las características personales, bloquea la creatividad y la expresión emocional, oprime las relaciones espontaneas y oprime el desarrollo de un pensamiento critico. El filosofo americano Chomsky a ese propósito sostiene que: "el verdadero aprendizaje tiene a que ver con el descubrimiento de la verdad, no con la imposición de la verdad oficial; esta ultima opción no conduce al desarrollo de un pensamiento critico y independiente"(Chomsky: 2013, p.29).

Desde aquí la necesidad de poner en evidencia como dentro de un contexto educativo de mirada libre, como lo que la Educación Libre propone, dirigido a un crecimiento integral sin vínculos ya establecidos, cambia radicalmente la construcción de la relación entre niños y niñas y sobretodo entre ellos y los adultos, poniendo al centro del proceso educativo el desarrollo emocional y el cuidado relacional, al fin de educar a una fortaleza interior fundada en una positiva relación con sí mismos y con los demás y en un desarrollo emocional libre.

5 Rebeca Wild, pedagoga de origen alemanes que en 1977 fundó, juntos a su marido, Mauricio Wild, el Centro educativo Pestalozzi llamado "Pesta". La estudiosa ha fallecido el 1 de noviembre del 2015, propio durante el desarrollo de este trabajo. Se agradece de los varios estímulos teóricos llegados desde los estudios y las reflexiones educativas de la pedagoga. 


\section{Una mirada al mundo de la Educación Libre}

Considero que la finalidad de la vida sea la felicidad;

ser feliz significa tener interés hacia algo.

La educación debería preparar para la vida.

En esto nuestra cultura no ba tenido logro.

Nuestra educación, política, economía llevan a la guerra.

(Neil:2012, p.46)

Estas las palabras de Alexander Neil, el fundador de Summerhill, la más famosa y exitosa escuela de educación libre europea, cuya idea básica se encuentra en la filosofía educativa de la mayoría de las realidades de Educación Libre, comprendidas las catalanas que se han analizados en esta investigación.

La Educación Libre, en efecto, no pretende ofrecer una educación para el mundo laboral, político o económico centrándose en objetivos como el saber competir, el logro profesional, la disciplina sin sentido critico, la directividad: “... casi todas las pedagogias han sido fundadas de bajo de una idea de hombre predefinida, de un modelo al que inspirarse para plasmar a través de la educación cada niño y cada niña. Esta creación de un prototipo recoge un autoritarismo implicito que cruza inexorablemente todas las filosofias educativas que se ban empeñado a hacer llegar a ser cada ser lo que, quien dirigía el proceso formativo, anhelaba o se sentia en deber de realizar"(Codello; Stella: 2011, p.29).

En contra de este planteamiento, la Educación Libre se coloca en el panorama pedagógico como una oportunidad para la actual sociedad en crisis de valores ofreciendo "otra" mirada basada en el respeto hacía los demás y basada en el amor; trabajando además por el crecimiento de niñas y niños en todas sus dimensiones, a partir de sus singularidades.

Las experiencias libres catalanas definen ${ }^{6}$, en este sentido, sus proyectos como espacios caracterizados por el respeto, la no violencia, el amor, el acompañamiento emocional, el respeto del ritmo personal, la libertad, el conocimiento de si mismo, o sea, lugares educativos en que se acompañe a los niños y niñas en el camino de su desarrollo hacia el descubrimiento de la vida, hacia el crecimiento respetuoso, hacia la conquista de la felicidad, hacia la formación de una identidad personal a partir de la aceptación de su ser interior; todas aquellas características revolucionarias, en un cierto sentido, para una sociedad en que impera la velocidad, el destaque emocional y la productividad. A este propósito dice Alexander Neil: "Desde el odio nace odio y desde el amor nace amor. Amor significa mantener una actitud positiva de aprobación, y esto es esencial en cualquier escuela. No se puede estar de la parte de los niños, y contemporáneamente castigarlos y asustarlos."(Neil: 2012, p.28)

6 Datos sacados por las preguntas n.17 y n.20 del cuestionario. 
Es difícil definir teóricamente ${ }^{7}$ qué es y qué representa la Educación Libre; en efecto es una forma de educación vivencial muy relacionada a la experiencia y a las prácticas específicas y no sigue un modelo ya establecido. Cada experiencia libre es, por tanto, una realidad diferente. Existen realidades que han sido originadas por iniciativa de educadores y educadoras, otras que son fundamentalmente proyectos de familias que contratan o no cuidadoras extras, y casi todas configuran un entramado de responsabilidades compartidas.

La mayoría de ellas son, por lo menos refiriéndonos a sus orígenes, una iniciativa de unas pocas familias con hijos pequeños. A veces el embrión de estas escuelas es un grupo de crianza, es decir, un grupo de madres y padres que se va encontrando regularmente durante el embarazo y los dos primeros años de vida del niño para compartir inquietudes, necesidades y vivencias. Posteriormente, algunas de estas familias, ante la dificultad de encontrar una propuesta educativa que consideren adecuada, optan por continuar con este espacio educativo que de manera espontánea ya se ha ido creando, y quizás abrirlo también a la incorporación de otros niños y niñas y sus familias.

Cada experiencia educativa "libre" o "alternativa" al sistema escolar formal, tiene sus propias peculiaridades y especificidades que la hacen llegar a ser especial y diferente de las otras, aunque la mayoría de dichas experiencias posee características comunes, como se lee en las siguientes palabras: “... respeto verdadero y profundo, tolerancia bacia a los sentimientos de niños y niñas, sensibilidad para sus necesidades, disponibilidad a aprender por ellos, honestidad y transparencia por parte de los educadores, de que la misma libertad definirá también la libertad de los pequeños"(Codello; Stella: 2011, p.35).

La Educación Libre, aunque no quiera definirse como un modelo bien estructurado, acoge en su propio 'hacer educativo' y en sus propias prácticas influencias que le llegan desde otros modelos educativos que han construido las propias metodologías en la idea de libertad educativa. Entre los estudiosos contemporáneos más importantes que han afectado positivamente la Educación Libre encontramos a Rebeca Wild, María Montessori, Ivan Illich, Alexander S. Neill, así como las ideas adoptadas de algunas escuelas democráticas o constructivistas.

Sin embargo cada experiencia educativa libre, al igual que cualquier grupo humano, tiene también sus limitaciones. A veces quien opta por planteamientos "alternativos" va cargado de expectativas inalcanzables, como la idea de que el espacio educativo que se desea crear para los hijos o hijas será perfecto, exento de situaciones conflictivas entre niños, niñas y adultos, y dará respuesta a todas sus necesidades.

Una elección de Educación libre es una pieza en la vida de un hijo o una hija y no toda su vida, su felicidad depende en la mayoría principalmente por la relación

7 Yo misma hasta que no he tenido la posibilidad de realizar una práctica en primera persona (año 2015) en el espacio de Educación Libre de la Caseta en Barcelona, no he podido comprender de forma plena la filosofía que aglutina la Educación Libre. 
con cada padre y madre, y también con si mismos, en la medida que irá a construir en su camino de desarrollo.

\title{
La importancia de los adultos: acompañantes, madres y padres
}

\author{
La sola idea que un niño pueda tener el control de su aprendizaje \\ y el derecho a elegir en ese sentido, \\ desbarajusta los convencimientos del adulto. \\ (Codello; Stella: 2011, p.40)
}

La educación para la libertad y en la libertad parte ya desde la construcción de la relación única y singular que se crea entre el adulto y el niño o la niña; dentro de esta existen unos elementos esenciales que los adultos tienen que considerar y, muchas veces, aprender. Uno de los más importantes lo plantea Maria Montessori en su pedagogía, o sea la clave del aprendizaje de la autonomía fundamentado en el ayudar niños y niñas a actuar por si mismos: "El pueblo que admite la servidumbre, que crea una ventaja del hombre el ser en todo servido y no, al revés, ayudado por el hombre, incluye como instinto el servilismo; en efecto fácilmente nos lanzamos a servir, como baciendo una zambullida en plena cortesía, en plena gentileza, en plena bondad. Sin embargo quien está servido recibe un daño para su independencia”. (Montessori: 2008, p.35)

Llegar a ser acompañantes en los proyectos de Educación Libre no es tan fácil, porque, a partir ya desde la actitud que presenta la pedagogía montessoriana, el mismo adulto tiene que destructurarse con respecto a las costumbres educativas que en su recorrido de vida, y en particular lo adquirido en lo profesional como educador, educadora, maestro, maestra, pedagogo y pedagoga; y repensar a su papel educativo hacia un proceso de crecimiento libre de niños y niñas. El acompañante entonces se convierte en facilitador de aprendizajes individuales, desarrollando lo más posible la competencia de ponerse a la escucha, de manera activa, de las necesidades de los pequeños, para acompañarlos en la realización de sus deseos.

Acompañar significa en gran parte, estar presente, disponible y observar. El acompañante organiza el tiempo y el espacio de manera que se favorezca el proceso de desarrollo autónomo de la criatura sin interferir, pero solo ofreciendo presencia y soporte, apoyo emocional y amor; su participación en las actividades infantiles llegan del placer de comunicarse con la criatura, sin intención de enseñar, acelerar, cambiar... El acompañante propone unas actividades o algún taller y los niños y las niñas son libres de elegir lo que más les interese.

En este sentido hemos preguntado a los veinte centros catalanes que componen la muestra de la presente investigación, las principales características personales y profesionales que debería poseer un adulto para ser un acompañante en un proyecto de Educación Libre ${ }^{8}$; la mayoría de los centros estudiados han contestado que no se valora tanto el titulo profesional como las actitudes personales demostradas

8 Datos obtenidos a partir de la pregunta n.11 del cuestionario.

Signos, Lajeado, ano 39, n. 1, p. 10-25, 2018. ISSN 1983-0378 
en la práctica, el recorrido personal y además características como el sentido critico y creativo, el deseo de aprender y de revisar las propias actitudes, la capacidad de comunicación horizontal (diálogo, escucha, observación, comunicación), el saber estar en silencio, la aceptación, el amor incondicional, la alegría, la no intervención, la humildad, la apertura, la ilusión, el respeto hacia uno mismo y hacia los demás.

Los centros investigados han explicitado también que la mayoría de los adultos que se acercan a un centro de Educación Libre, ya conocen esta realidad educativa y tienen algún conocimiento en el tema. En bastantes ocasiones provienen de un recorrido de estudio o de experiencia en educación, en psicología, en masaje o en terapia infantil, pero también hay quien llega desde el mundo del arte y del teatro; en cualquier caso cada adulto o adulta que se implica en un proyecto de Educación Libre no solo posee un saber teórico, sinó además su historia personal, sus conocimientos y su propio ser y, por entonces, elige de experimentarse en un contexto que exige una puesta en juego personal total.

Todas las realidades de Educación Libre, se puede decir que están caracterizadas por el hecho de no ser espacios masificados; en efecto suelen tener una relación numérica entre adulto, niños y niñas más o menos de 1/5-6. Cada niño o niña construirá entonces una relación con el acompañante con el que trabajará en las diferentes actividades, teniendo la posibilidad de disfrutar de un espacio relacional singular y de todo el tiempo que necesitará para vincularse con él o con ella.

Este espacio simbólico es muy favorable a la construcción de una relación educativa significativa, punto básico para la Educación Libre, fundada en la confianza y el crecimiento conjunto, a partir del respeto de la necesidad de niños y niñas de vivir en espacios tranquilos y que no provoquen posible dispersión.

La Educación Libre se pone en contra a la tendencia del modelo escolar donde en los pocos metros cuadrado de una aula un sólo adulto tiene que tratar con una media entre los 25 y 30 niños y niñas, no llegando jamás a construir una verdadera relación singular y afectando mucho a la calidad relacional: "...los muros de la clase y de los edificios escolares, hechos como prisiones, restringen la abertura mental de los profesores e impiden de ver los elementos esenciales de la educación. Su trabajo toma en cuenta solo la parte del niño que se encuentra arriba del cuello; y necesariamente, la parte vital del niño, la emotiva, queda para ellos como territorio extranjero". (Neil: 2012, p.51)

Otra clave de logro educativo de la Educación Libre se encuentra en la postura que los y las acompañantes adoptan a la hora de actuar educativamente.

Desde los datos de la pregunta n.12 del cuestionario, se puede evidenciar como los mayores porcentajes en sentido negativo (nada, poco, discreto), se encuentran en las conceptualizaciones como la dirección, el autoritarismo, la imposición, el control, el juicio, la disciplina como adiestramiento, que se pueden considerar muy lejanas a las prácticas presentes en un proyecto de Educación Libre; al revés se cuenta un porcentaje alto, en sentido positivo (bastante y mucho), con respecto a posturas cuales compartir emociones, escucha, amor, cuidado, respetosa de los deseos, respetosa del ritmo personal, responsabilidad, acompañamiento, recursos 
de apoyo, colaboración. Llama la atención la respuesta "hermanos/as mayores" a la que la mayoría de la muestra (17) ha contestado negativamente, signo de gran importancia otorgada a la Familia y a la separación, entre los acompañantes y las componentes y los familiares, con respecto a los roles en la área psico-afectiva. Una puntuación muy elevada de la muestra (17) ha obtenido también la afirmación "interesada a los saberes personales que llevan (niños y niñas)" que pone en evidencia el extremo respeto para la infancia, vista y considerada como portadora de conocimientos, saberes y, por qué no, sabiduría. Desde el análisis se puede decir con seguridad que la dirección tomada por los acompañantes de las experiencias de Educación Libre catalanes en las posturas llevadas en adelante es sin duda de cercanía afectiva, del cariño, de presencia y del acompañamiento emocional.

La educación de las emociones es un pilar fundamental, junto a la relación educativa, de la Educación Libre, en efecto la principal responsabilidad de los adultos implicados es la de acompañar los niños y las niñas en sus procesos de crecimiento a través del descubrimiento y del conocimiento de las propias emociones en relación a los acontecimientos cotidianos y de las relaciones que cada día se construyen.

"...una de las características más fascinantes de un acompañante de las escuelas libres y democráticas, es la capacidad de observar los niños mientras actúan, de escuchar sus discursos, coger su expectativas, sostener sus frustraciones, estimular las preguntas y la búsqueda de respuestas, revisar soluciones y conocimientos adquiridos, individuar por tanto sus etapas evolutivas". (Codello; Stella: 2011, p.44) 
Figura 8: Gráfico datos - pregunta n.12.

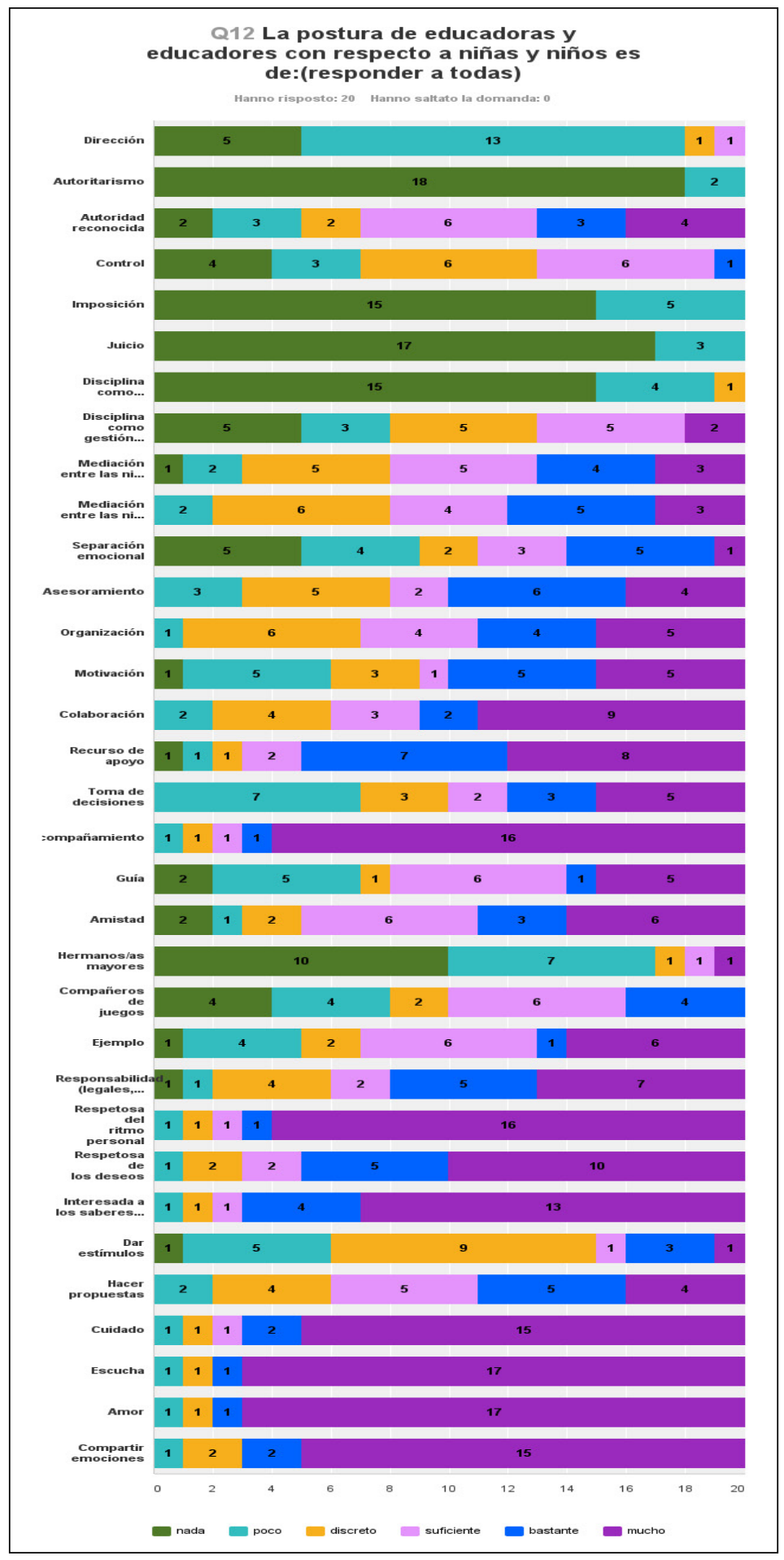

Fuente: cuestionario. 
En la gestión de los espacio de Educación Libre el papel de las familias resulta ser fundamental. Estas están plenamente implicadas en la vida de los centros $\mathrm{y}$ en el proceso educativo de los niños y niñas; en este sentido se consideran el núcleo principal del crecimiento y de la educación de los hijos y hijas. Muchos proyectos educativos están gestionados por las mismas familias que se juntan para ofrecer recorridos de crecimientos diferentes a los escolares y organizan los espacios como si fueran una gran familia; otros, gestionados educativamente por acompañantes, consideran padres y madres como elementos imprescindibles en el desarrollo de los procesos educativos de niños y niñas y crean con ellos una estrecha y constante colaboración para asegurar las mejores condiciones para una sana y armoniosa educación.?

En este sentido padres y madres son considerados "los adultos más importantes de la vida de niños y niñas" ${ }^{10}$ y "los responsables primeros de la educación y desarrollo de los niños" ${ }^{11}$ con los cuales crean vínculos de vital importancia para la formación de su seguridad emocional, base para todo su posterior aprendizaje.

En las realidades de Educación Libre se habla entonces de corresponsabilidad educativa y participación conjunta para que se asegure confianza, compromiso y la consideración del proyecto como un espacio propio y no como un servicio ${ }^{12}$. La responsabilidad compartida entre la familia y los acompañantes por un lado vincula la primera a desarrollar la práctica de estar en presencia ${ }^{13}$ y "una necesaria coherencia entre la manera de acompañar en el hogar y en el proyecto" ${ }^{14} \mathrm{y}$ por otro lado consolida la importancia de una mirada educativa unitaria, especialmente en el conocimiento del contexto psicoafectivo del niño y de la niña: "el niño trae consigo toda una familia con sus características y eso tiene efectos directos sobre él" 15 .

La familia resulta necesaria al buen desarrollo de hijos e hijas, y mucho más cuando se coordina y trabaja conjuntamente y en armonía con el centro educativo, considerándolo como una prolongación del hogar y de la familia misma: "La educación de la niña y el niño es posible a través del entramado de las relaciones que se generan en su ambiente. El hecho de que los dos agentes, familia y escuela, se encuentren en colaboración es fundamental para un desarrollo sano y una formación integral en las infancias" ${ }^{16}$.

9 http://www.educaciolliure.org consultado en Octubre 2015.

10 La Caseta, respuesta a la pregunta n.13 del cuestionario.

11 El Roser, respuesta a la pregunta n.13 del cuestionario.

12 Moixaina, respuesta a la pregunta $\mathrm{n} .13$ del cuestionario.

13 Práctica educativa en femenino trabajada por el Pensamiento de la Diferencia.

14 Agambar, respuesta a la pregunta n.13 del cuestionario.

15 La Serra, respuesta a la pregunta n.13 del cuestionario.

16 Petit Molinet, respuesta a la pregunta n.13 del cuestionario.

Signos, Lajeado, ano 39, n. 1, p. 10-25, 2018. ISSN 1983-0378 
Las familias desarrollan también algunas actividades relacionadas con la gestión del centro mismo y su ayuda es muy importante para la vida del mismo, porque los acompañantes no pueden encargarse de todas las tareas existentes.

Con respecto a la situación catalana, los datos de la investigación evidencian como los cargos mayores tomados por las familias se refieren a gestiones administrativas y organizativas y como, al revés, la parte más pedagógica se queda a cargo de los acompañantes. Esta resulta una buena y armoniosa distribución de tareas entre los adultos y adultas que forman parte de los proyectos de Educación Libre.

\section{El valor de las relaciones en la Educación Libre}

La Educación Libre considera la relación educativa como uno de sus puntos básicos con la finalidad de asegurar un desarrollo armonioso del ser y de la identidad personal en los niños y las niñas.

La primera cosa que se aprende en los contextos educativos libres se centra en instaurar relaciones con los demás, acompañantes o compañeros y compañeras que sean. Cada niña y cada niño experimenta la relación con el otro y la otra, construye su identidad, explora su carácter y aprende a vivir teniendo en cuenta a los demás y respetándoles.

Podemos considerar la relación como un particular espacio de crecimiento y de confrontación; es un lugar simbólico en el que cada uno o una construye, a partir de sí mismo y sí misma, con el otro y la otra; gracias a estas características cada relación nace y se desarrolla como única y diferente de cualquier otra.

Dentro de una relación y, sobre todo gracias a ella, cada uno o una crece, evoluciona. Y por ello, resulta positivo desarrollar la capacidad de tejer varias y diferentes relaciones para que cada una de ellas deje un nudo importante para el camino personal.

La primera relación que experimenta es con el adulto, una relación, para la Educación Libre, basada sobre todo en la presencia, el soporte, el amor y la aceptación total al ser del niño o de la niña. Dentro de la relación las criaturas se sienten libres de ser sí mismas y encuentran su propio espacio especial y único con el adulto, junto al cual crecen y se desarrollan dejando algo de sí mismos y de su saber y tomando algo del adulto, en una dinámica circular.

De los centros de Educación Libre catalanes consultados, han contestado por lo que se refiere a la tipología de relación educativa que se construye entre los/ las acompañantes y niños y niñas que para la mitad de ellos ( 9 centros) la relación educativa es asimétrica, aunque con reconocimiento de los roles y niveles diferentes; la otra mitad (8 centros) considera que se trata de una relación simétrica, o sea al mismo nivel, y también con un reconocimiento de roles diferentes ${ }^{17}$.

17 Datos obtenidos de la pregunta n.45 del cuestionario. 
Desde este análisis se evidencia que en ambos los casos la relación educativa que se desarrolla en los centros de Educación Libre analizados tiene en consideración como elemento fundamental la diferenciación, por lo que respecta al rol, que poseen adultos y pequeños. Cada uno por su parte pone sí mismo en la relación que se construye y que llega a ser educativa porque circularmente se transforma en continuación dejando los mismos actores transformados, madurados, cambiados, contaminados de las características del otro.

Siempre con respecto a la relación educativa entre los y las acompañantes y niños y niñas, la mayoría de los centros declaran de no encontrar una diferencia en la relación entre acompañantes hombres o mujeres y niños y niñas ${ }^{18}$. Los centros consideran que la relación no depende tanto del sexo sinó de la postura y de las características personales de los y las acompañantes. Aseguran que gracias a la observación se evidencia la tipología específica del acercamiento que tienen los niños y las niñas con el otro sexo, en base a lo que han experimentado especialmente en la familia con sus propios padres o madres; en este sentido dice el centro libre El Roser: "Algunos niños y niñas prefieren tomar de referente a un educador y otros a una educadora. Hemos visto que esto no está en relación con el sexo, es decir los niños no siempre eligen a los educadores como referente ni viceversa. A veces nos encontramos con niñas que rechazan la figura masculina como acompañante. Creemos que estas relaciones se basan mucho en el tipo de relación que han tenido los niños y niñas con otros adultos de referencia (familia) durante los primeros años" $"$.

De gran importancia desde el punto de vista educativo resultan también las relaciones que se construyen entre niñas y niños; estas también llegan a ser espacios propios de crecimiento y de desarrollo con características diferentes de las que se instauran con los adultos pero con una gran carga emocional.

Las relaciones entre "los pares" llevan los mismos niños y las mismas niñas a ponerse en juego en primera persona en la dinámica, a aprender a respetar al otro y su espacio vital, a obtener aprendizajes desde las experiencias compartidas y a construir conjuntamente una dinámica vivencial, a resolver si surge algún conflicto; destacamos las palabras de Xantala (otra realidad libre): "El objetivo es que la relación entre ellos sea de igual a igual y que autónomamente puedan ir llegando a acuerdos en sus juegos, resolviendo sus conflictos y ir vinculándose desde un lugar sano donde cada uno sea amado por lo que es" ${ }^{20}$.

Uno de los elementos más delicado en la dinámica de las relaciones hace referencia a las emociones vividas en las relaciones entre niños y niñas ${ }^{21}$, porque desde su propia interpretación e interiorización se pueden desarrollar experiencias

18 Datos obtenidos de la pregunta n.46 del cuestionario.

19 El Roser - respuesta a la pregunta n.46 del cuestionario.

20 Xantala - respuesta a la pregunta n.48 del cuestionario.

21 Datos obtenidos de la pregunta n.48 del cuestionario. 
positivas o negativas que influenciarán también las relaciones siguientes; como considera el espacio libre de Gatzara por ejemplo, con respecto a su actuación: "La relación tiene que ser respetuosa, es decir que se sientan bien en la relación. Favorecemos la comunicación, explicarnos las cosas, las que nos gustan y las que no" 22.

La postura asumida por la mayoría de los centros de Educación Libre de Cataluña analizados es la de dejar que niños y niñas encuentren su propia vía en las relaciones que construyen, también si estas son conflictuales, aprendiendo así la práctica del respeto hacia los demás y la construcción de un pensamiento crítico propio.

Nos dice el centro libre de Espai Aigua a ese propósito que su postura en las dinámicas relacionales entre niñas y niños es la de "Dejar que encuentren sus propios recursos, que se enfrenten a los conflictos y a la búsqueda de las soluciones que mejor les convenga; que encuentren y pacten las normas de convivencia que ellos puedan asumir. Respeto profundo por el otro, ayudando como educadores a analizar, relativizar y ser críticos" ${ }^{23}$.

El tema de las relaciones es muy fascinante porque cada una de ella lleva consigo un mundo emocional único. Para el pensamiento de la diferencia sexual las relaciones son un tejido vivo de intercambios y no una masa estable e inmóvil, y que permiten la atención a lo singular (Jourdan: 2001, p.113); así es como se interpretan desde la Educación Libre.

\section{Conclusión}

La opinión publica es siempre más critica hacia la educación formal y resulta en aumento el numero de familias que deciden recorridos y formas alternativas a la escuela tradicional para la educación, la instrucción y la formación de los propios hijos y hijas. Hoy en día se asiste a numerosas reformas o cambios respecto a la educación formal pero todavía no es colmada la carencia de la educación respecto a la libertad de ser y de elegir, a la educación a las relaciones, a la educación emocional, a la educación fundada en el amor.

Los educandos tienen a que ver con programas escolares viejos y no renovados y con prácticas educativas que no dejan a los y las jóvenes espacios para auto-educarse y crecer en manera no constrictiva.

La Educación Libre intenta desarrollar experiencias y prácticas libres para poder ofrecer nuevos recorridos educativos fundados en un pensamiento y en una postura que no parte del objetivo para alcanzar pero de las necesidades de cada niño y cada niña.

Su trabajo educativo encuentra unos pilares fundamentales, como hemos visto en este trabajo de investigación, en la política de las relaciones; se crece gracias y en las relaciones positivas y también conflictivas que cuotidianamente se construyen,

22 Gatzara - respuesta a la pregunta n.48 del cuestionario.

23 Aigua - respuesta a la pregunta n.48 del cuestionario.

Signos, Lajeado, ano 39, n. 1, p. 10-25, 2018. ISSN 1983-0378 
desde aquí la total puesta en juego de adultos, niños y niñas en la tensión dinámica y relacional caracterizada por una asimetricidad circular en que la relación de autoridad es presente pero en continua transformación y distribución entre los actores de la relación.

Esta situación de disparidad educativa favorece el intercambio constante de los papeles de educando/a y educador/dora, decretando, en tal sentido, un verdadero reconocimiento del niño y de la niña como portadores de una fresca sabiduría y de una experiencia viva.

El valor de las relaciones educativas es muy alto y favorece la experimentación de estas en espacios protegidos, como los de un centro educativo, no puede que ayudar a niños y niñas a ponerse en juego y a aprender a relacionarse en primer lugar con sí mismo y sí misma y después con los demás, a descubrir los propios limites, a vivir los conflictos, a reconocer sin frustración un valor o una sabiduría en los demás, a dar sentido a las relaciones y sobretodo a sentirse libres de ser sí mismos y sí mismas.

La Educación Libre desarrolla la práctica de las relación educativa cotidianamente demostrando que una educación integral, armoniosa y libre es posible también gracias al coraje y al empeño de los adultos implicados en el proceso educativo, dispuestos a repensar a su propia postura para cambiarla y a ponerse a la escucha de los pequeños para aprender de ellos.

Aqui reside entonces el arte educativa: en el saber tomar medida de la acción de ayudo al desarrollo de la personalidad infantil.

(Montessori: 2008, p.134)

\section{Bibliografía}

AA.VV. (2013). La (A) en la pizarra. Escritos anarquistas sobre educación. Madrid: LaMalatesta Ed.

Alves, R. (2003). La scuola che ho sempre sognato. Bologna: EMI.

Bakunin, M. (2009). La libertà degli uguali. Milano: Elèuthera.

Blanco, N. (2001). Educar en Femenino y en Masculino. Madrid: Akai.

Cavarero, A., Restaino, F. (2002). Le filosofie femministe. Milano: Bruno Mondadori.

Chomsky, N. (2013). La (Des) Educacion. Barcelona: CRITICA.

Codello, F. (2005). La buona educazione. Esperienze libertarie e teorie anarchiche in Europa da Godwin a Neill. Milano: Franco Angeli.

Codello, F., Stella, I. (2011). Liberi di imparare. Firenze: Terra Nuova. 
Codello, F. (2005). Vaso, creta o fiore? Né riempire, né plasmare ma educare. Lugano: La Baronata.

Codello, F. (2009). Né obbedire né comandare. Milano: Elèuthera.

Contreras Domingo, J., Arnaus i Morral, R. (2015). Educacio alternativa: Moviments creatius cap a una obertura pedagogica. Temps de Educacion, $n .48$. Barcelona: Universitat de Barcelona.

Dewey, J. (2004). Democrazia e Educazione. Firenze: Sansoni.

Freire, P. (1973). L'educazione come pratica della libertà. Milano: Arnoldo Mondadori.

Freire, P. (2004). Pedagogia dell'autonomia. Saperi necessari per la pratica educativa. Milano: EGA ed Gruppo Abele.

Freire, P. (2010). La pedagogia degli oppressi. Milano: EGA ed Gruppo Abele.

García Lastra, M., Calvo Salvador, A., Susino Rada, T. (2008). La mujeres cambian la educación. Madrid: Narcea.

Holt, J. (1977). Bisogni e diritti dei fanciulli. Roma: Armando.

Illich, I. (1972). Descolarizzare la società. Milano: Mondadori Editore.

Illich, I., El Rebozo (2013). Tramas de aprendizaje convivencial. Cuadernos para la imaginación. Oaxaca México: El Rebozo.

Korczak, J. (1996). Come amare il bambino. Milano: Luni.

Korczak, J. (2012). Il diritto del bambino al rispetto. Roma: Edizioni dell'Asino.

Montessori, M. (2008). Educare alla Libertà. Milano: Arnoldo Mondadori.

Montessori, M. (2012). La mente del bambino. Milano: Garzanti.

Neill, S. A. (1956). Questa terribile scuola. Firenze: La Nuova Italia.

Neill, S. A. (2012). I ragazzi felici di Summerbill. Milano: Red.

Piussi, A. M., Mañeru Méndez A. (2006). Educación, nombre común femenino. Barcelona: OCTAEDRO.

Tomasi, T. (1973). Ideologie libertarie e formazione umana. Firenze: La Nuova Italia.

Vigilante, A., Vittoria, P. (2011). Pedagogie della liberaz̧ione. Foggia: Edizioni del Rosone.

Wild, R. (2011). Educar para ser. Vivencias de una escuela activa. Barcelona: Herder. 